\title{
ANÁLISE EXPERIMENTAL DE PERFIS DE VELOCIDADE EM RISER POR MEIO DE TÉCNICA DE STEREO PIV
}

\author{
C. B. STREY ${ }^{*}$, J. UTZIG ${ }^{1,2}$, R. K. DECKER ${ }^{1}$, H. F. MEIER ${ }^{1}$ \\ ${ }^{1}$ Universidade Regional de Blumenau, Departamento de Engenharia Química \\ ${ }^{2}$ Universidade Federal de Uberlândia, Faculdade de Engenharia Mecânica \\ e-mail: caroline-bosco@hotmail.com
}

\begin{abstract}
RESUMO
O comportamento do escoamento gás-sólido num riser foi estudado por meio da determinação experimental de perfis de velocidade de ambas as fases. Os experimentos foram realizados na região próxima à entrada de catalisador de uma unidade experimental de leito fluidizado circulante a frio em escala de laboratório. O riser apresenta diâmetro interno de $0,104 \mathrm{~m}$, altura de 2,4 m e região de medição cilíndrica feita em acrílico. Os experimentos foram conduzidos em duas condições operacionais: velocidade superficial de gás, de 5,6 e $8,3 \mathrm{~m} / \mathrm{s}$, a uma taxa mássica constante de sólidos de 8,6 g/s, correspondente a razões de carga aproximadas de 180 e $120 \mathrm{~g} / \mathrm{m}^{3}$, respectivamente. A obtenção dos perfis de velocidade foi realizada em três alturas ao longo do riser por meio da técnica óptica, minimamente intrusiva, de Velocimetria por Imagem de Partícula Estereoscópica (Stereo PIV). A partir dos resultados obtidos observou-se um comportamento qualitativo muito semelhante para ambas as condições operacionais, tanto nos experimentos referentes à fase gasosa, quanto nos experimentos referentes à fase sólida. Notou-se maior concentração de sólidos na parede oposta à alimentação de sólido, mais evidente para a velocidade superficial de gás de 5,6 m/s, por meio de observações visuais do escoamento na região de entrada do sólido.
\end{abstract}

\section{INTRODUÇÃO}

O processo de craqueamento catalítico fluidizado (FCC) é caracterizado pela quebra de grandes moléculas de hidrocarbonetos na presença de partículas de catalisador num leito que escoa no sentido ascendente (riser). O objetivo é converter frações pesadas de petróleo em frações mais leves, que compõem produtos de maior valor comercial (SADEGHBEIGI, 2000).

O riser corresponde a um leito de fluidização rápida, no qual se observa alta taxa de arrastamento dos sólidos pelo gás, sendo este um aspecto interessante em processos que envolvem reações rápidas. No entanto, quando há a necessidade de recirculação de sólidos para compensar as perdas do leito, estes são chamados de leitos fluidizados circulantes (KUNII e LEVENSPIEL, 1991; NITZ e GUARDANI, 2008).

O comportamento fluidodinâmico gássólido pode interferir no rendimento da unidade de FCC, uma vez que exerce influência sobre as reações de craqueamento. Deste modo, os fenômenos que se desenvolvem no interior do riser precisam ser bem conhecidos para que seja possível o aprimoramento contínuo do processo.

Neste sentido, diversos estudos foram realizados com o intuito de melhor compreender e modelar o escoamento gássólido encontrado em risers, os quais aplicaram ferramentas computacionais e experimentais. 
Estudos experimentais realizaram medidas de velocidade ou de distribuição de sólidos em unidades experimentais de leito circulante a frio, utilizando técnicas sofisticadas de medição, em geral pontuais (PANTZALI et al., 2013; VAN ENGELANDT et al., 2007; BHUSARAPU, AL-DAHHAN e DUDUKOVIĆ, 2006; GODFROY, LARACHI e CHAOUKI, 1999; VAN DEN MOORTEL et al., 1998), sendo poucos deles aliados a simulações numéricas (GAO et al., 2009; DE WILDE et al., 2005).

Em relação a estudos de simulação, alguns se dedicaram com maior ênfase ao conhecimento da fluidodinâmica (HODAPP, 2009; MARINI, 2008; BASTOS, 2005; HUILIN et al., 2003; RIBEIRO, 2001) enquanto outros buscaram aplicar processos de transferência de calor e reações químicas de craqueamento (LOPES, 2012; ROSA, 2002).

Diante deste contexto, o presente estudo apresenta a obtenção e a análise de dados experimentais de perfis de velocidade próximos à região de entrada de catalisador num riser de uma unidade experimental a frio de leito fluidizado circulante. $\mathrm{O}$ objetivo consiste em desenvolver uma base de dados experimentais para aplicação em estudos de validação, consolidando, deste modo, uma unidade experimental projetada para aplicação em estudos na região de entrada de risers.

\section{MATERIAIS E MÉTODOS}

É apresentada a seguir uma descrição da unidade experimental, bem como da técnica aplicada para a obtenção de dados. Além disso, são abordados alguns aspectos específicos do procedimento experimental referente a cada fase de interesse, sendo definido ainda o planejamento experimental.

\subsection{Descrição da Unidade Experimental}

Com o intuito de promover estudos experimentais, na região de entrada de risers de FCC em um sistema com leito circulante em regime de fluidização rápida, foi projetada e construída a Unidade Experimental de Leito Circulante (UE-LC) no Laboratório de Verificação e Validação da Universidade Regional de Blumenau. A UE-LC permite a avaliação do comportamento dos campos de velocidade do gás e do sólido em diferentes condições operacionais, o que é de grande importância para o entendimento dos efeitos da alimentação de catalisador no riser. Para representar o escoamento no interior de um riser de FCC, utiliza-se ar à temperatura ambiente como fase gasosa e o próprio catalisador de FCC como fase sólida.

A unidade, cuja representação tridimensional é apresentada na Figura 1 constitui-se basicamente de um riser (2), um ciclone (3), um alimentador de sólidos (4) e um exaustor centrífugo (8) para promover o escoamento com depressão. O riser apresenta diâmetro interno de $104 \mathrm{~mm}$, altura aproximada de $2,4 \mathrm{~m}$ e foi planejado de modo a permitir adaptações geométricas, bem como visualização do escoamento no seu interior, contando com partes desmontáveis e tubos em material acrílico que permitem a observação do escoamento e a medição de campos de velocidade por meio da técnica não intrusiva de Velocimetria por Imagem de Partícula Estereoscópica (Stereo PIV). Na entrada de ar (1) do riser, outro duto de acrílico de $0,5 \mathrm{~m}$ de comprimento e $90 \mathrm{~mm}$ de diâmetro interno fica acoplado em um joelho de $90^{\circ}$. O catalisador é introduzido pelo alimentador de sólidos, sendo que sua entrada no sistema se dá por meio de um tubo em Y, formando um ângulo de $45^{\circ}$ em relação ao eixo do riser. A separação primária entre ar e catalisador é realizada por um ciclone de voluta em aço inoxidável que, alinhado com o reservatório do alimentador, o reabastece com o catalisador separado.

Para garantir uma boa separação do catalisador remanescente na corrente de gás que deixa o ciclone, esta é encaminhada a um 
Figura 1 - Representação tridimensional da Unidade Experimental de Leito Circulante.

1 - Entrada de ar

2 - Riser

3 - Ciclone

4 - Alimentador de sólidos

5 - Filtro de mangas

6 - Painel elétrico

7 - Computador

8 - Exaustor

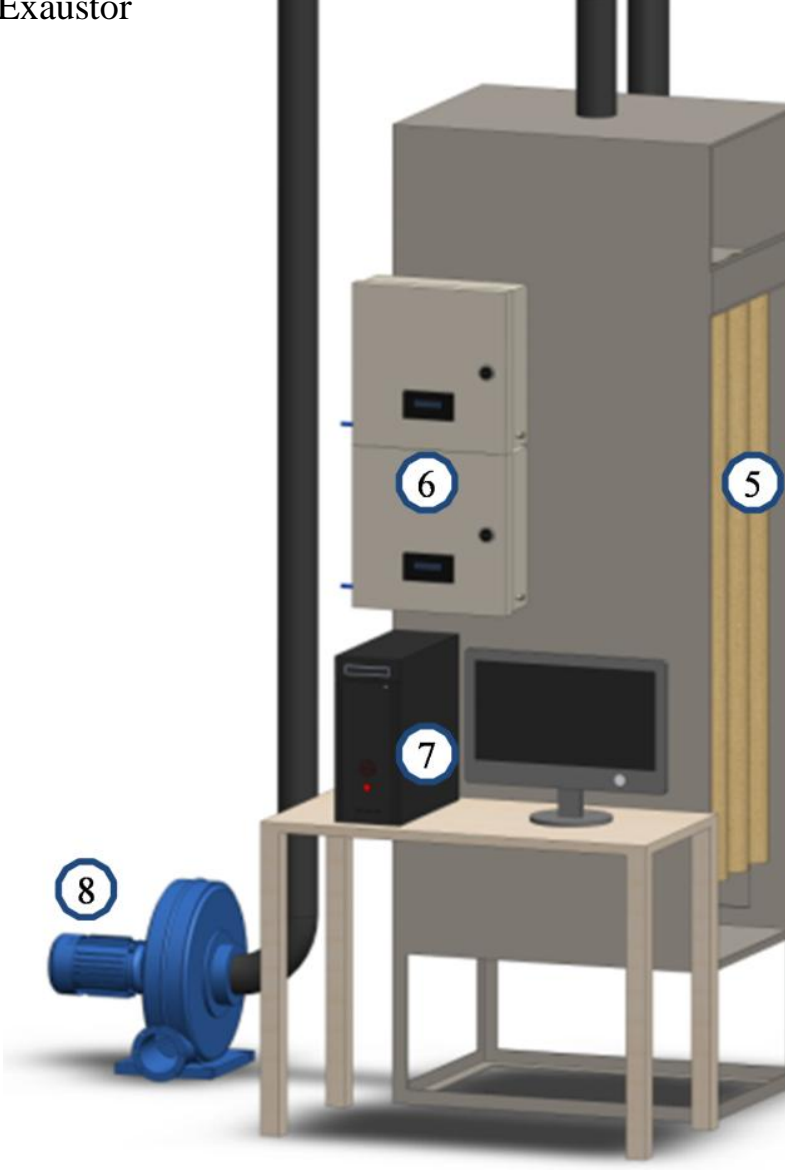

filtro de mangas (5). O escoamento da unidade é promovido por um exaustor centrífugo. $\mathrm{O}$ exaustor fica localizado no final da linha, mantendo o sistema sob vácuo parcial.

Para obtenção de medidas macroscópicas no sistema, dispõe-se de um tubo de Pitot conectado a um manômetro diferencial, bem como um sensor para a leitura de temperatura e outro para pressão estática, localizados na tubulação anterior ao filtro de mangas. Com base nas medidas obtidas pelo tubo de Pitot e demais sensores, um software instalado no computador (7) monitora e controla a vazão de gás na unidade pela atuação no exaustor. Por meio da interface do software, é possível ajustar manualmente a potência do exaustor e do alimentador, cujos inversores de frequência encontram-se instalados no painel elétrico (6).

\subsection{Técnica de Velocimetria por Imagem de Partícula}

A Velocimetria por Imagem de Partícula (PIV) é uma técnica óptica, minimamente intrusiva, utilizada para a 
obtenção de medidas instantâneas de campos de velocidade de escoamentos. O princípio da técnica consiste na visualização da trajetória das partículas presentes no meio que, percorrendo determinados espaços em tempos conhecidos, permitem a determinação dos vetores de velocidade.

Neste trabalho foi utilizada a técnica de Stereo PIV (3D), que consiste numa extensão do conceito 2D. Por meio desta é possível medir as três componentes de velocidade simultaneamente, bem como corrigir automaticamente distorções que se devem a efeitos de curvatura, que se faz necessária em medições do escoamento num duto cilíndrico, por exemplo. Neste caso, duas câmeras posicionadas em diferentes ângulos capturam a imagem das partículas iluminadas no escoamento em estudo. Enquanto que uma câmera só é capaz de medir a projeção do movimento das partículas perpendicular ao eixo óptico, a utilização de duas câmeras permite a observação do real deslocamento das partículas no interior do volume de medição (LAVISION, 2001). Um esquema de comparação entre os métodos de medição 2D e 3D é apresentado na Figura 2.

Figura 2 - Esquema para comparação entre as técnicas de PIV 2D e 3D.

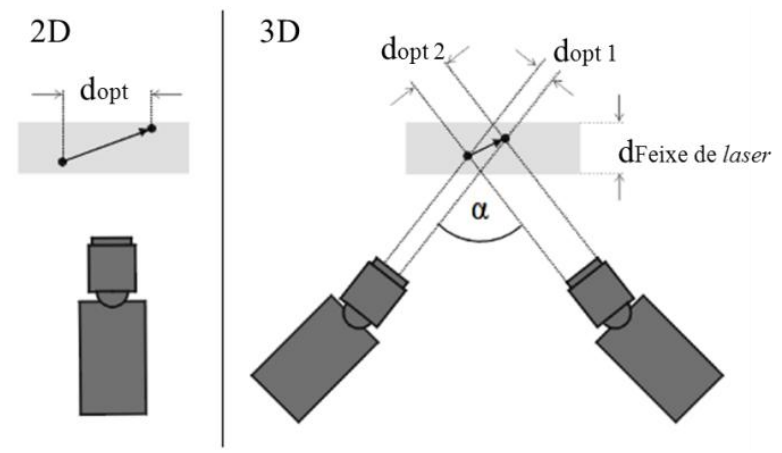

Fonte: Adaptado de LAVISION (2002).

\subsubsection{Partículas Traçadoras}

Para quantificar a fidelidade dos traçadores em relação ao escoamento turbulento pode-se utilizar como parâmetro o número de Stokes (St), definido conforme a
Equação (1), o qual corresponde à razão entre o tempo de reação da partícula $\left(\tau_{s}\right)$, dado pela Equação (2), e o tempo característico do escoamento $\left(\tau_{\text {esc }}\right)$, dado pela Equação (3).

$$
\begin{aligned}
& \text { St }=\frac{\tau_{\mathrm{s}}}{\tau_{\mathrm{esc}}}, \\
& \tau_{\mathrm{s}}=\frac{\rho_{\mathrm{s}} \mathrm{d}_{\mathrm{s}}{ }^{2}}{18 \mu_{\mathrm{g}}}, \\
& \tau_{\mathrm{esc}}=\frac{\mathrm{D}}{\mathbf{v}_{\mathrm{g}}} .
\end{aligned}
$$

Partindo deste critério de análise, partículas que apresentam St $<0,1$ se comportam como partículas de fluido, podendo ser utilizadas como traçadoras (BRANDON e AGGRWAL, 2001).

Nos experimentos foram utilizadas partículas de catalisador de FCC para a determinação dos perfis de velocidade da fase sólida e partículas de catalisador de FCC moído para representar a fase gasosa, uma vez que, por apresentarem menor diâmetro, as partículas moídas passam a atender ao critério estabelecido pelo número de Stokes.

Para a determinação da granulometria do material particulado alimentado nos experimentos de medição de velocidade, da fase gasosa e da fase sólida, foi utilizado um sistema de medição de distribuição granulométrica de partículas, modelo Mastersizer 2000, com unidade de dispersão Hydro 2000MU, fabricados pela empresa Malvern. Este equipamento mede a intensidade da luz disseminada quando um feixe de laser passa através de uma amostra de partículas dispersas e analisa tais dados para calcular o tamanho das partículas, criando um padrão de distribuição.

Os resultados das análises realizadas para as amostras das partículas de catalisador 
de FCC e de catalisador de FCC moído são apresentados, respectivamente, na Figura 3 (a) e na Figura 3 (b).

Figura 3 - Distribuição granulométrica de partículas: (a) catalisador de FCC e (b) catalisador de FCC moído.
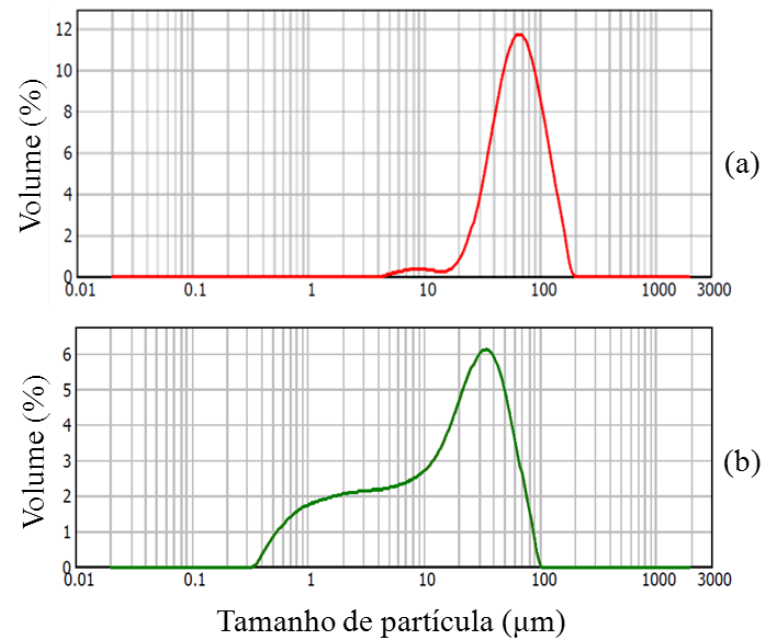

A partir da análise de distribuição granulométrica são obtidos alguns diâmetros característicos como d $(0,1), \mathrm{d}(0,5)$ e d $(0,9)$. O valor de $\mathrm{d}(0,5)$ é definido como o diâmetro onde metade da população fica abaixo deste valor. Do mesmo modo, $10 \%$ da distribuição fica abaixo do valor de $\mathrm{d}(0,1)$ e $90 \%$ da distribuição fica abaixo do valor de $\mathrm{d}(0,9)$. Além dos valores característicos, outros diâmetros de natureza estatística, são fornecidos pela análise, sendo estes o $\mathrm{D}[3,2]$ (diâmetro médio Sauter) e o D[4,3] (diâmetro médio de De Brouckere). O diâmetro $\mathrm{D}[3,2]$ representa a média dos diâmetros de esferas de área superficial igual a das partículas reais e é mais sensível à presença de partículas finas na distribuição de tamanhos. O diâmetro $\mathrm{D}[4,3]$, por sua vez, representa a média dos diâmetros de esferas de volume igual a das partículas reais e é mais sensível à presença de grandes partículas na distribuição de tamanhos (MALVERN, 2012). Os valores dos diâmetros citados, referentes a cada uma das amostras de partículas analisadas, são apresentados na Tabela 1.
Tabela 1 - Diâmetros das amostras de partículas.

\section{Partícula}

Diâmetro Catalisador de Catalisador de FCC $(\mu \mathrm{m}) \quad$ FCC moído $(\mu \mathrm{m})$

\begin{tabular}{lcc}
$\mathrm{d}(0,1)$ & 32,279 & 1,281 \\
$\mathrm{~d}(0,5)$ & 64,273 & 16,750 \\
$\mathrm{~d}(0,9)$ & 119,231 & 52,346 \\
$\mathrm{D}[3,2]$ & 51,314 & 4,021 \\
$\mathrm{D}[4,3]$ & 70,431 & 22,125 \\
\hline
\end{tabular}

Determinando-se o valor de St com base no diâmetro das partículas de catalisador de FCC moído, cuja amostra apresentou $\mathrm{d}_{\mathrm{s}}=\mathrm{D}[3,2]=4,021 \mu \mathrm{m}, \quad$ considerando-se a massa específica de $\rho_{\mathrm{s}}=1400 \mathrm{~kg} / \mathrm{m}^{3}$ e demais características do escoamento $\left(\mu_{\mathrm{g}}=1,8 \times 10^{-5}\right.$ $\mathrm{kg} / \mathrm{m} \cdot \mathrm{s}, \mathrm{D}=0,104 \mathrm{~m}$ e $\mathbf{v}_{\mathrm{g}}=8,3 \mathrm{~m} / \mathrm{s}$ ) obteve-se St $=0,006$, ou seja, St $\ll 0,1$, justificando-se assim a utilização de catalisador de FCC moído para a representação do escoamento do gás. Levando-se em conta as mesmas características do escoamento e massa específica dos sólidos, para partículas de catalisador de $\mathrm{FCC}, \mathrm{D}[3,2]=51,314 \mu \mathrm{m}$, obteve-se o valor de St $=0,9$, portanto, $\mathrm{St}>$ 0,1 , o que indica que causam interferência no escoamento, sendo úteis para a observação da influência da entrada de sólidos no sistema estudado.

\subsection{Metodologia da Experimentação}

Por meio de experimentos físicos, buscou-se obter perfis dos componentes de velocidade da fase sólida e da fase gasosa em pontos distribuídos no raio do riser, aplicando-se a técnica de Stereo PIV.

Os procedimentos da experimentação foram realizados posteriormente às devidas calibrações do manômetro diferencial do tubo de Pitot e do sistema de Stereo PIV. De maneira simplificada, as etapas iniciais da experimentação consistem em definir a velocidade superficial de gás desejada no riser, aguardar sua estabilização e alimentar o 
sistema com partículas traçadoras de acordo com a fase de interesse.

Nos experimentos de medida de velocidade da fase gasosa, as partículas de catalisador de FCC moído foram inseridas no sistema a partir da entrada de ar utilizando-se um alimentador de sólidos, modelo Particle Blaster 100 do fabricante LaVision, o qual utiliza ar comprimido para fluidizar o material particulado presente no seu reservatório, garantindo uma alimentação uniforme das partículas traçadoras. Nas medições de velocidade da fase sólida, as partículas de catalisador de FCC foram inseridas no riser por meio do alimentador de sólidos que compõe a unidade experimental.

Em cada medição foram capturados 1000 pares de imagens, a uma frequência de aquisição de aproximadamente $7,5 \mathrm{~Hz}$. Os perfis foram obtidos pela média dos 1000 pares de imagens.

\section{RESULTADOS E DISCUSSÕES}

Os resultados dos experimentos, apresentados mais adiante, correspondem aos perfis dos componentes da velocidade média de escoamento em pontos distribuídos no raio do riser, com as respectivas flutuações de velocidade em cada ponto apresentadas na forma de barras. Em relação à experimentação aplicada para ambas as fases, é importante ressaltar que técnica de Stereo PIV realiza as medições com base nas partículas inseridas no escoamento, ou seja, quando avalia-se a fase gasosa tem-se somente as partículas traçadoras no sistema e quando avalia-se a fase sólida tem-se somente as partículas de catalisador.

Os perfis de velocidade foram medidos em três alturas do riser, com intervalos de 0,3 $\mathrm{m}$ entre as alturas de medição, sendo a primeira localizada a $0,35 \mathrm{~m}$ do ponto de entrada de catalisador. As linhas onde foram obtidas as velocidades na direção radial referem-se ao eixo $\mathrm{Z}$ da geometria (Figura 4), variando entre -50 e $50 \mathrm{~mm}$, sendo que o feixe de laser foi posicionado de modo a iluminar um plano $\mathrm{YZ}$, passando pelo centro do riser. Os locais de aquisição de dados estão ilustrados na Figura 4, para facilitar a visualização dos resultados.

Figura 4 - Esquema simplificado de posicionamento das linhas e alturas de medição.

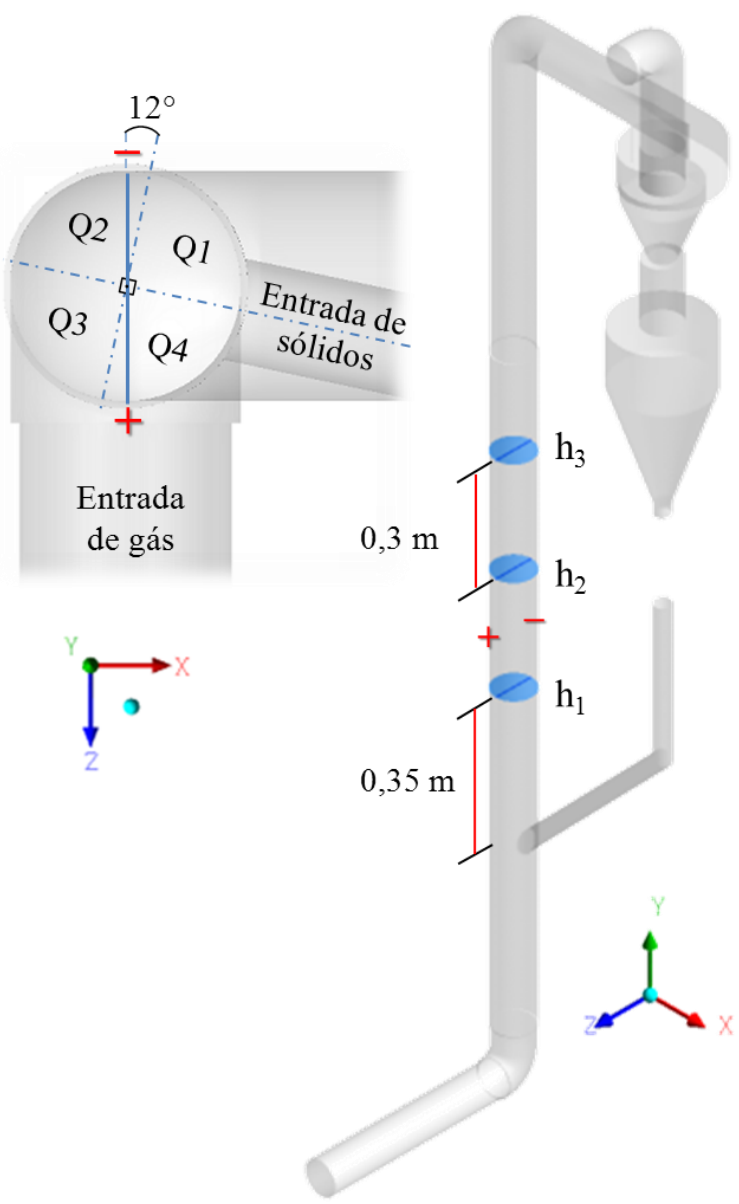

$\mathrm{Na}$ Figura 5, para cada altura de medição, são apresentados os perfis de velocidade correspondentes à fase gasosa para a condição de velocidade superficial de gás no riser de $5,6 \mathrm{~m} / \mathrm{s}\left(\mathbf{v}_{\mathrm{g}, 1}\right.$, símbolos em cor preta) e de $8,3 \mathrm{~m} / \mathrm{s}\left(\mathbf{v}_{\mathrm{g}, 2}\right.$, símbolos em cor azul $)$. A disposição dos perfis está de acordo com o que se observa ao longo da altura (h) do riser, com resultados da altura $\mathrm{h}_{1}$ na posição inferior, $h_{2}$ na posição intermediária e $h_{3}$ na posição superior. 
Figura 5 - Perfis dos componentes de velocidade média e RMS média para a fase gasosa.

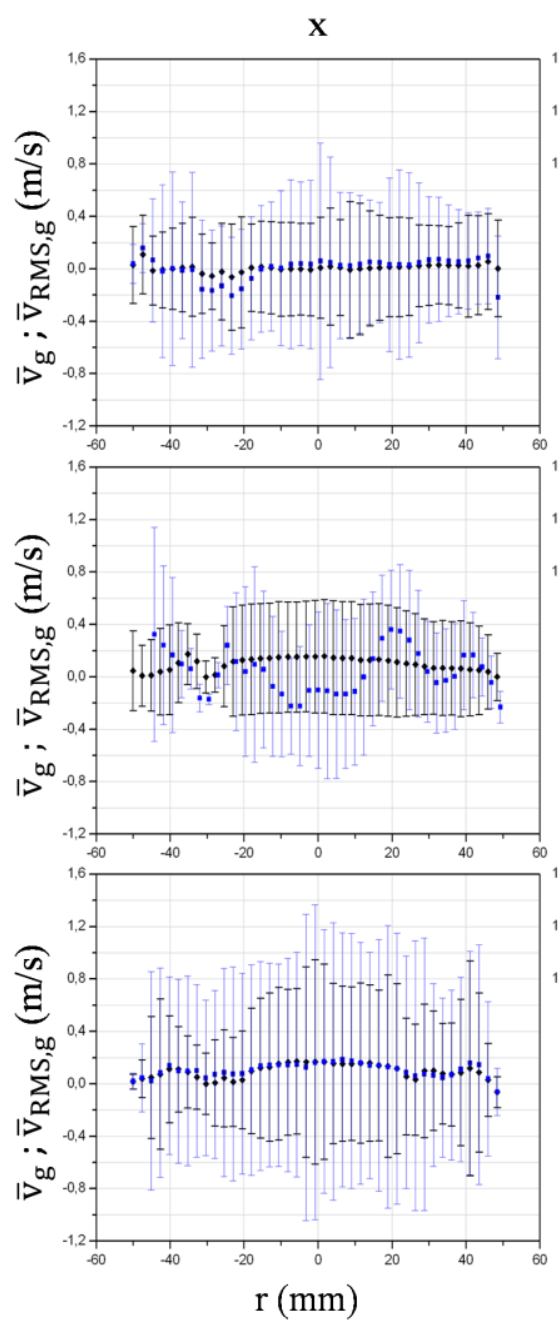

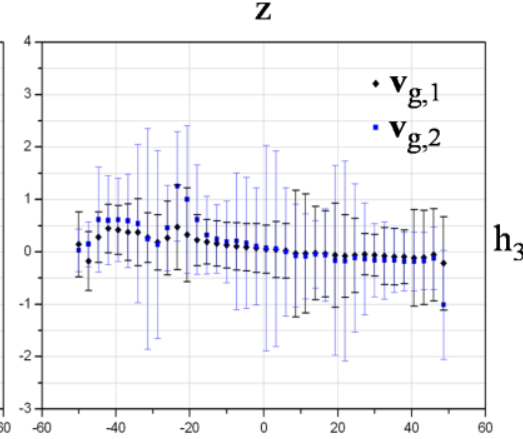
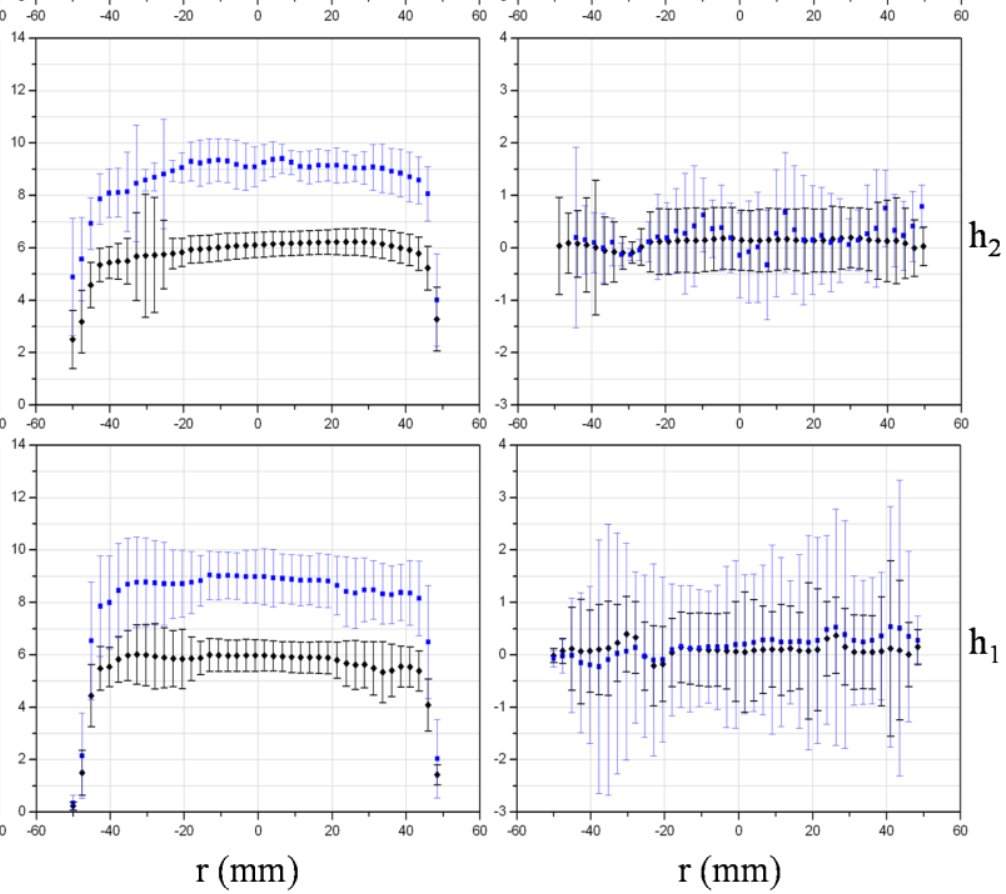

Pela análise da Figura 5 observa-se em todas as alturas de medição que as magnitudes de velocidade dos componentes em $\mathrm{X}$ e em $\mathrm{Z}$ são menores se comparadas às componentes em Y, apresentando, no entanto, maiores amplitudes em relação às flutuações. As flutuações se mostraram maiores para a condição de velocidade superficial do gás mais elevada. Além disso, nota-se que, para ambas as condições operacionais, as velocidades em $X$ e em $Z$ se mantiveram muito próximas, ao contrário do que se observa na direção $\mathrm{Y}$, onde as condições podem ser facilmente distinguidas. A similaridade entre as velocidades radiais $\mathrm{e}$ suas magnitudes próximas a zero, demonstra

que o escoamento é majoritariamente axial nestas posições. Em relação aos perfis da direção Y, não é possível identificar influência da curva localizada logo após a entrada de ar, constatando-se que o escoamento se desenvolve rapidamente, visto que não observam-se mudanças significativas nos perfis ao longo da altura do riser. Conforme a posição do plano de medição, verifica-se que os componentes em $\mathrm{Y}$ correspondem à velocidade axial e os componentes em $\mathrm{Z}$ à velocidade radial, tendose em X a componente estereoscópica, cujos valores de velocidade são menos precisos.

A Figura 6, apresenta os perfis referentes à fase sólida nas condições de 
velocidade superficial de gás no riser, $\mathbf{v}_{\mathrm{g}, 1} \mathrm{e}$ $\mathbf{v}_{\mathrm{g}, 2}$, e vazão mássica constante de $8,6 \mathrm{~g} / \mathrm{s}$ de catalisador de FCC, que correspondem a razões de carga aproximadas de 180 e 120 $\mathrm{g} / \mathrm{m}^{3}$, respectivamente.

Na Figura 6 nota-se um comportamento semelhante aos obtidos para a fase gasosa, apesar de se observar menores flutuações na fase sólida. No entanto, ao contrário da fase gasosa, cujo escoamento se desenvolve rapidamente, no caso da fase sólida é possível notar variação dos perfis ao longo da altura do riser. $\mathrm{Na}$ altura $\mathrm{h}_{1}$ as velocidades axiais mais baixas, que ocorrem nas posições radiais entre 0 e $50 \mathrm{~mm}$, podem ser atribuídas a um balanceamento da quantidade de movimento devido à alimentação a montante. Assim que as partículas entram no riser, retiram energia das regiões Q2 e Q3 (conforme Figura 4), fazendo com que o gás acelere na metade oposta (Q1 e Q4). Conforme o escoamento se desenvolve, as partículas são redistribuídas e as maiores velocidades passam a ser observadas, em $\mathrm{h}_{1}$, nas regiões Q2 e Q3, uma vez que a conservação da quantidade de movimento deve ser mantida.

Este fenômeno também se evidencia nos comportamentos opostos observados em $\mathrm{h}_{1}$ e $\mathrm{h}_{3}$, para a velocidade radial. Valores positivos se alternam entre as duas metades do riser, indicando que as partículas movemse de um lado a outro. Observa-se na altura $\mathrm{h}_{1}$

Figura 6 - Perfis dos componentes de velocidade média e RMS média para a fase sólida.
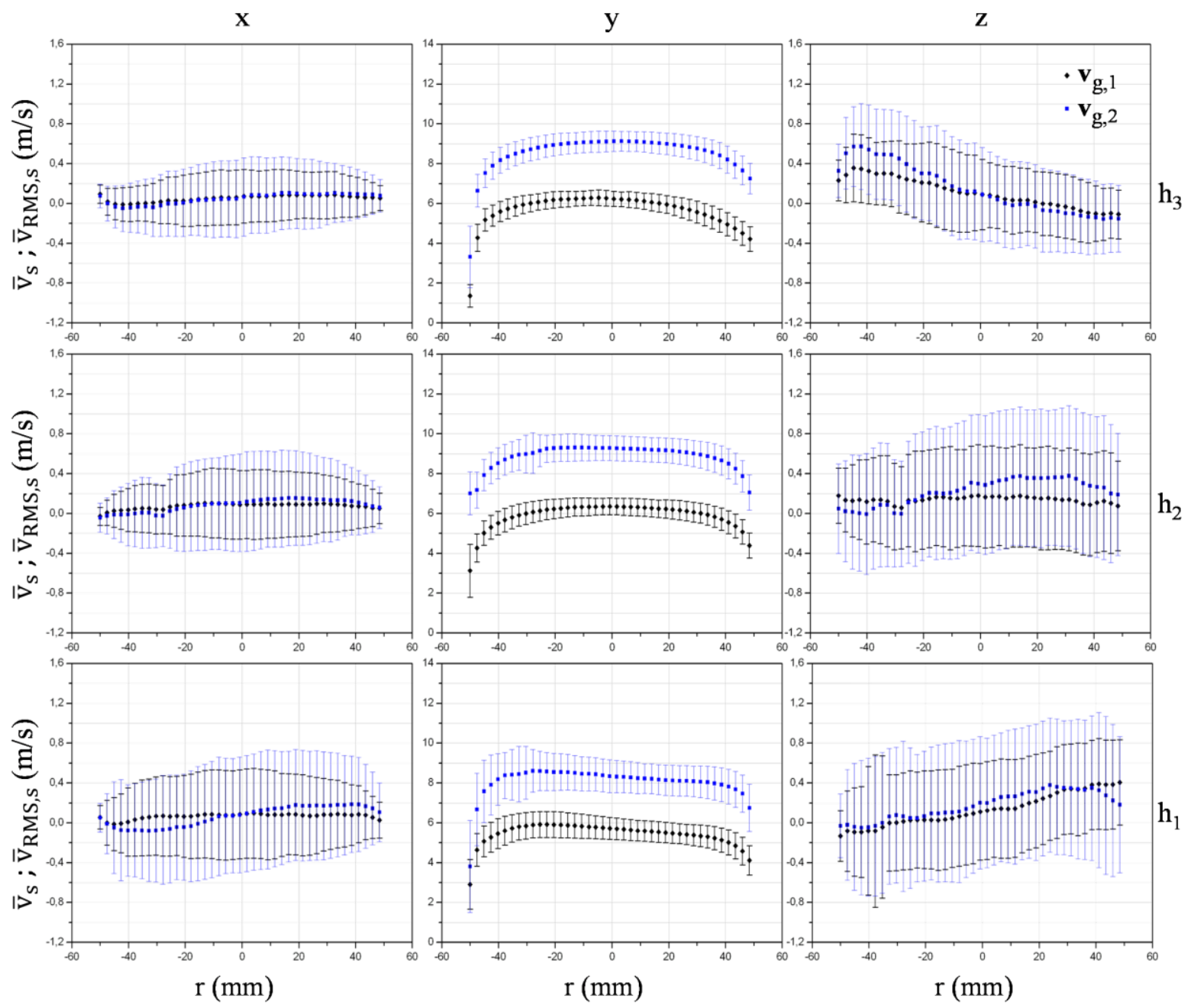
uma movimentação das partículas do centro para as paredes, mais evidente na direção $\mathrm{Z}$. Este mesmo efeito, com menor intensidade, pode também ser notado na direção $X$ para essa altura, bem como na direção $\mathrm{Z}$ para a altura $\mathrm{h}_{2}$, quando considerada a condição $\mathbf{v}_{\mathrm{g}, 2}$. $\mathrm{Na}$ altura $h_{3}$ verifica-se uma movimentação das partículas contrária à observada na altura $\mathrm{h}_{1}$ para a direção $\mathrm{Z}$.

Com o intuito de ilustrar as condições em que os experimentos que incluíram a fase sólida foram conduzidos, na Figura 7 é apresentada a região de engaste do riser com a entrada de catalisador, para cada uma das velocidades superficiais de gás no riser.

Figura 7 - Região de engaste do riser com entrada de catalisador, para $\mathbf{v}_{\mathrm{g}, 1}$ e $\mathbf{v}_{\mathrm{g}, 2}$.

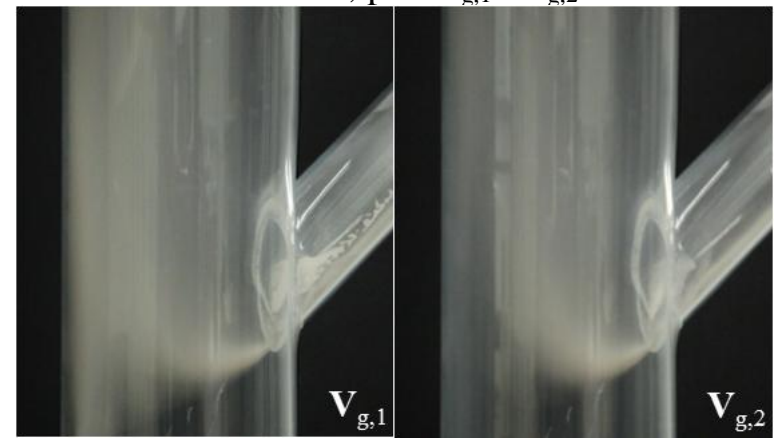

Para a velocidade $\mathbf{v}_{\mathrm{g}, 1}$ é possível perceber a maior concentração de sólidos na parede oposta e na região logo abaixo a sua entrada. Para a velocidade $\mathbf{v}_{\mathrm{g}, 2}$ nota-se uma diminuição da concentração de sólido na parede oposta, onde o gás é capaz de arrastar o sólido com mais facilidade.

\section{CONCLUSÕES}

Foi possível a realização de um estudo visando uma melhor compreensão do escoamento gás-sólido encontrado em risers, mediante a obtenção de perfis de velocidade pela aplicação da técnica de Stereo PIV. Deste modo, constituiu-se uma base experimental para aplicação em estudos de validação de modelos matemáticos fluidodinâmicos.
Diante dos perfis obtidos para a direção axial, foi possível observar o desenvolvimento do escoamento para ambas as fases ao longo da altura do riser. O escoamento monofásico é predominantemente axial, enquanto que as partículas de catalisador deslocam-se radialmente, especialmente na região mais próxima à entrada de sólidos.

\section{NOMENCLATURA}

D : Comprimento característico do escoamento $(\mathrm{m})$

$\mathrm{d}_{\mathrm{p}}$ : Diâmetro da partícula da fase sólida $(\mu \mathrm{m})$

St : Número de Stokes

$\mathbf{v}_{\mathbf{g}}$ : Velocidade média da fase gasosa $(\mathrm{m} / \mathrm{s})$

$\mu_{\mathrm{g}}$ : Viscosidade dinâmica da fase gasosa $(\mathrm{kg} / \mathrm{m} \cdot \mathrm{s})$

$\rho_{\mathrm{s}} \quad$ : Massa específica da fase sólida $\left(\mathrm{kg} / \mathrm{m}^{3}\right)$

$\tau_{\text {esc }}$ : Tempo característico do escoamento (s)

$\tau_{\mathrm{s}}:$ Tempo de reação da partícula (s)

\section{REFERÊNCIAS}

BAStOS, J. C. S. C. Simulação do escoamento gás-sólido em um duto cilíndrico vertical em leito fluidizado rápido aplicando a técnica CFD. Campinas, 2005. 82p. Dissertação (Mestrado em Engenharia Química) - Universidade Estadual de Campinas.

BHUSARAPU, S., AL-DAHHAN, M. H., DUDUKOVIĆ, M. P. (2006). Solids flow mapping in a gas-solid riser: Mean holdup and velocity fields. Powder Technology, v. 163, n. 1-2, p. 98-123, abr. 2006.

BRANDON, D. J.; AGGARWAL, S. K.; A numerical invertigation of particle deposition on a square cylinder placed in a channel flow. Aerosol Science and Technology, v. 34, p. 340-352, 2001. 
DE WILDE, J., VAN ENGELANDT, G., HEYNDERICKX, G. J., MARIN, G. B. (2005). Gas-solids mixing in the inlet zone of a dilute circulating fluidized bed. Powder Technology, v. 151, n. 1-3, p. 96-116, 2005.

GODFROY, L., LARACHI, F., CHAOUKI, J. Position and Velocity of a Large Particle in a GadSolid Riser using the Radioactive Particle Tracking Technique. The Canadian Journal of Chemical Engineering, v. 77, p. 253 - 261, abr. 1999.

HODAPP, M. J. Modelagem e simulação de um leito fluidizado: um estudo comparativo. Campinas, 2009. 113p. Dissertação (Mestrado em Engenharia Química) - Universidade Estadual de Campinas.

HUILIN, L., GIDASPOW, D., BOUILLARD, J., WENTIE, L. Hydrodynamic simulation of gas-solid flow in a riser using kinetic theory of granular flow. Chemical Engineering Journal, v. 95, n. 1-3, p. 1-13. Jan. 2003.

KUNII, D.; LEVENSPIEL, O. Fluidization engineering. 2.ed. Stoneham: Reed Publishing, 1991.

LAVISION (DE). FlowMaster: manual. Göttingen: LaVision GmbH, 2002.

LAVISION (DE). FlowMaster: advanced PIV systems for quantitative flow field analysis. Göttingen: LaVision GmbH, 2001.

LOPES, G. C. Estudo computacional da dinâmica do escoamento reativo em risers industriais de FCC. Campinas, 2012. 108p. Tese (Doutorado em Engenharia Química) Universidade Estadual de Campinas.

NITZ, M.; GUARDANI, R. Fluidização gássólido: fundamentos e avanços. Revista Brasileira de Engenharia Química, p. 2024, dez. 2008.
PANTZALI, M. N., LOZANO BAYÓN, N., HEYNDERICKX, G. J., MARIN, G. B. (2013). Three-component solids velocity measurements in the middle section of a riser. Chemical Engineering Science, 101, p. 412423, jul. 2013.

RIBEIRO, D. C. Implementação de modelo de fechamento em risers de FCC através de código comercial CFD. Campinas, 2001. 80p. Dissertação (Mestrado em Engenharia Química) - Universidade Estadual de Campinas.

ROSA, L. M. Simulação de reações químicas e consumo de calor em risers. Campinas, 2002. 76p. Dissertação (Mestrado em Engenharia Química) - Universidade Estadual de Campinas.

SADEGHBEIGI, R. Fluid catalytic cracking handbook: design, operation and troubleshooting of FCC facilities. 2. ed. Houston: Gulf Publishing Company, 2000.

VAN DEN MOORTEL, T., AZARIO, E., SANTINI, R., TADRIST, L. Experimental analysis of the gas-particle flow in a circulating fluidized bed using a phase Doppler particle analyzer. Chemical Engineering Science, v. 53, n. 10, p. 18831899, jan. 1998.

VAN ENGELANDT, G., DE WILDE, J., HEYNDERICKX, G. J., MARIN, G. B. Experimental study of inlet phenomena of inclined non-aerated and aerated Y-inlets in a dilute cold-flow riser. Chemical Engineering Science, v. 62, n. 1-2, p. 339-355, 2007.

\section{AGRADECIMENTOS}

Os autores agradecem ao apoio financeiro da Petrobras, mediante o termo de cooperação 0050.0070334.11.9, que permitiu o desenvolvimento deste trabalho. 